\title{
THE BIOCHEMICAL PROCESS OF LUBRICANT FILM FORMATION IN JOINT REPLACEMENT
}

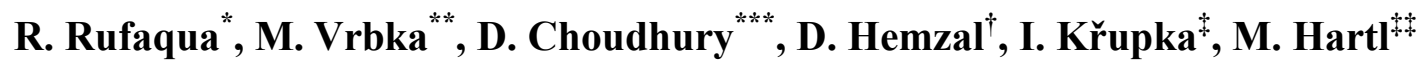

Abstract: The main objective of this study is to clarify the biochemical reactions of the formation of the lubricating film between the head and the cup of the joint replacement. The chemical changes taking place within the proteins of synovial fluid constitute after joint replacements need to be observed. Film formation is also dependent on the $\mathrm{pH}$ of the solution and static and rolling conditions, viscosity, concentration of the solution and other mechanical properties of the total environmental condition of joint replacement. The chemical composition of synovial fluid is predominantly considered to explain the development of film on the ball. The experiment was conducted in ball-on-cup configuration in a hip joint simulator, where the ball was made of cobalt chromium molybdenum alloy and the cup was of optical glass. The used proteins albumin, $\gamma$ globulin and bovine serum were the reactants of this process. To analyze the products of the reaction, Raman spectroscopic method was used to observe the changes among proteins. After the analysis of the Raman spectra, it is assumed heterogeneous catalysis process could be taken place on the metal ball during chemical reaction. The results could have significant information regarding lubricating film development within the joint replacement.
\end{abstract}

Keywords: synovial fluid, film formation, Raman spectroscopy, bio-tribology, tribo-chemistry

\section{Introduction}

The biochemical composition of synovial fluid is very complex. Differences occurred in synovial fluid compositions for the normal knee joint, patients without replacement of joint, patients with primary arthroplasties and patients with revisions of total hip and knee arthroplasties (Galandáková et al., 2017). Protein, which is a naturally occurring component of synovial fluid (Hills et al., 2003), varies its concentration during artificial joint replacement occurs. Particularly the concentrations of such constituents as Hyaluronic acid, proteins and phospholipid concentrations varied widely in patients undergoing these processes (Mazzucco et al., 2004). The thickness of the lubricant film on hip prostheses could be the contribution of particular proteins (Nečas et al., 2016). The protein concentrations of Bovine Serum Albumin (BSA), albumin and $\gamma$-globulin influence the frictional behaviour of the CoCr femoral head (Duong et al., 2012). To explain the biochemical reactions of lubricating film formation, Spectroscopy could be an excellent method to describe the chemical bonding among protein constituents. Raman spectra are able to show that a correlation between the level of phospholipids and proteins exists (Depciuch et al., 2016). Therefore, it is high time we clarified the chemical reaction of synovial fluid components due to the film formation of the joint replacement.

* Risha Rufaqua, PhD student. Faculty of Mechanical Engineering, Brno University of Technology, Technicka 2896/2, 616 69, Brno, Czech Republic. Risha.Rufaqua@vut.cz

** Assoc. Prof. Martin Vrbka, PhD. Faculty of Mechanical Engineering, Brno University of Technology, Technicka 2896/2, 616 69, Brno, Czech Republic. Martin.Vrbka@vut.cz

*** Dipankar Choudhury, PhD. Nano Mechanics and Tribology Laboratory, Department of Mechanical Engineering, University of Arkansas, Fayetteville, AR, USA. dc020@uark.edu

$\dagger$ Dušan Hemzal, PhD. Department of condensed Matter Physics, Faculty of Science, Masaryk University, Kotlarska 2, 61137 Brno, Czech Republic. hemzal@monoceros.physics.muni.cz

* Prof. Ivan Křupka, PhD. Faculty of Mechanical Engineering, Brno University of Technology, Technicka 2896/2, 616 69, Brno, Czech Republic. Ivan.Krupka@vut.cz

\# Prof. Martin Hartl, PhD. Faculty of Mechanical Engineering, Brno University of Technology, Technicka 2896/2, 616 69, Brno, Czech Republic. Martin.Hartl@vut.cz 


\section{Methods}

\subsection{Pendulum hip simulator}

The Pendulum hip simulator is found as an unprecedented and unique bio-tribological instrumentation that replicates 'flexion and extension' of artificial hip joints condition, alongside their real geometry, body temperature and load. It is also capable of measuring a real-time velocity profile, average friction coefficients and a viscous effect (the indicator of lubrication film formation). The simulator is composed of a base frame with an acetabular cup and a swinging pendulum with a femoral head. Electromagnetic motors are employed to drive the pendulum allowing a continuous motion in the flexion-extension plane (Choudhury, 2015 Vrbka, 2015 and Nečas, 2017).

\subsection{Ball on cup configuration}

To clarify the reaction processes in artificial joints, we are considering the metal femoral heads reaction with particular proteins. The experiment was conducted on ball-on-cup configuration. To explain the effect of the chemical reaction within metal hip prosthesis head, CoCrMo ball $(28 \mathrm{~mm})$ was used as per ASTM F-75. The cup was used that is made up of optical glass.

\subsection{Protein lubricants}

For this experiment, the contact was lubricated by Bovine Calf Serum, albumin and $\gamma$-globulin to achieve the result from Raman Spectroscopy. 25\% Bovine Calf Serum was used. $28 \mathrm{mg} / \mathrm{ml}$ of albumin solution and $11 \mathrm{mg} / \mathrm{ml}$ of $\gamma$-globulin were prepared with Phosphate buffer solution. Thus, during the experiment, the pendulum hip joint simulator set up was used to observe the tribological effect for 5-6 minutes to obtain the chemical effect on the ball of proteins as the lubricants.

\subsection{Raman spectroscopy}

Raman spectroscopy provides information on the chemical structure of compounds through identification and analysis of functional groups and also expresses the change of bond polarities of compounds. Since water is one of the components of the biological materials, Raman spectrum does not interfered with water. To detect conformational changes and structural differences of biomolecules on a metal surface, surfaceenhanced Raman scattering (SERS) is very useful (Zou, 1998, Shanmukh, 2006, Han 2009 and Depciuch, 2016). Therefore, using RENISHAW in Via Raman spectrometer, spectra were obtained for protein solutions before and after tribological experiments in the simulator.

\section{Results}

The spectra found on metal ball for all lubricants were quite different from the spectra of without-test liquid of the same protein, the reason of the difference could be due to the crystalline form of the protein on metal ball (see Fig. 1).

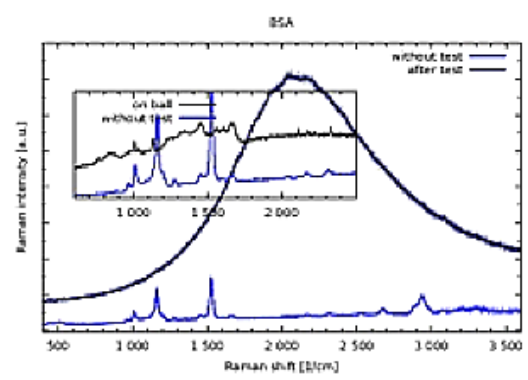

(a)

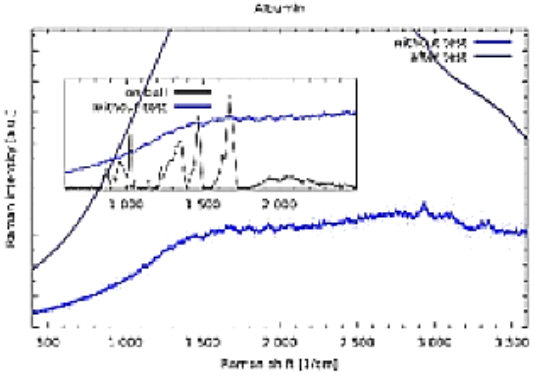

(b)

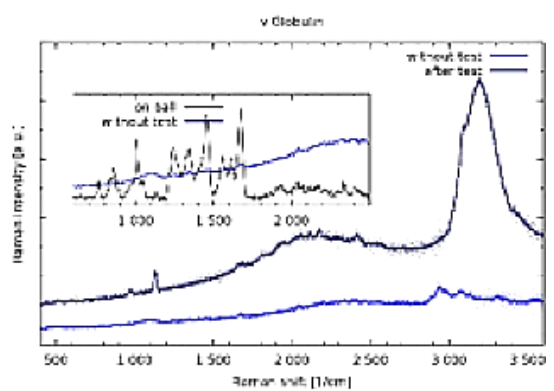

(c)

Fig. 1: Raman fingerprint spectra of solutions without tribological test and after tribological experiment together with Raman spectra of the film formed on CoCrMo ball with lubricants (a) BSA, (b) albumin, (c) y-globulin. 
There could be also the existence of chemisorbed transition state within protein lubricants and metal surfaces. After experiment protein liquid spectra for BSA and albumin have similarities but did not show appropriate spectra, although after-test $\gamma$-globulin shows well defined peaks. Most probably the $\beta$-sheet structure of $\gamma$-globulin is affected upon chemical reactions. The $\alpha$-helical structure of BSA and albumin become distorted.

The formed film structures on CoCrMo ball surface for BSA, albumin and $\gamma$-globulin were observed during the Raman experiment in order to investigate the biochemical influence of protein lubricant on metal surface.

(a)
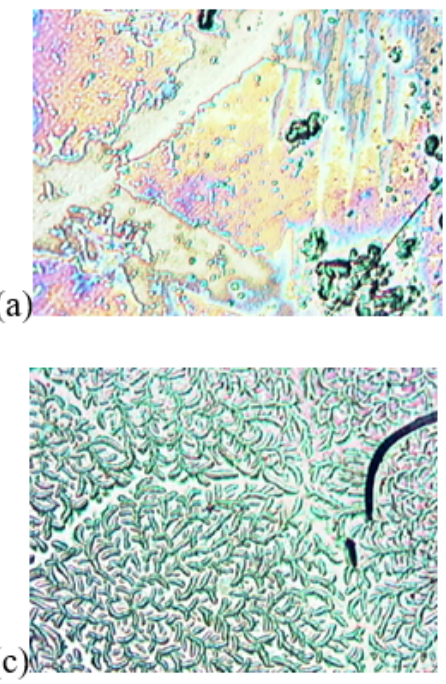

(d)

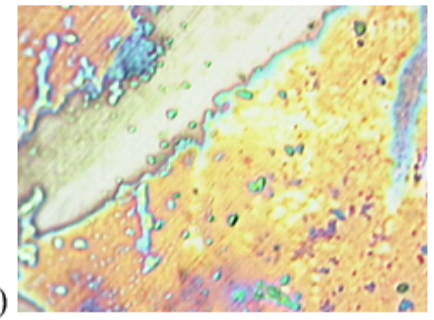

(b)

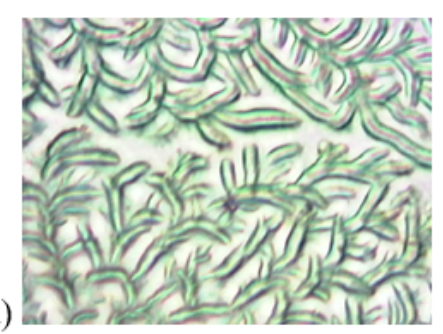

(e)
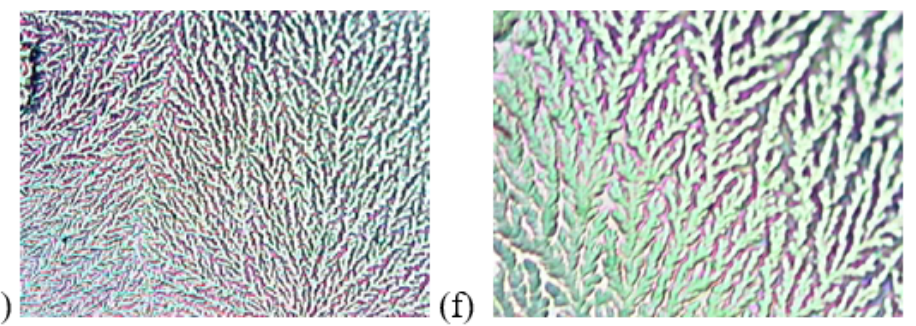

Fig. 2: Film formed viewed with Raman microscope on ball by respectively (a) BSA and (b) BSA enlarged view, (c) albumin and (d) albumin enlarged view and (e) $\gamma$-globulin and $(f) \gamma$-globulin enlarged view. The horizontal size of panels is $180 \mu \mathrm{m}(a, c, e)$ and $70 \mu \mathrm{m}(b, d, f)$.

The different structures of the formed film produced by different proteins are clearly visible from the Fig. 2 . Thus, these observations can also play an important role in biochemical explanation of adsorption taking place on the metal surface by protein molecules during film formation.

\section{Conclusion}

Therefore, to describe the condition of joint replacement and to increase the longevity of the replaced area, the chemical changes taking place in the proteins of synovial fluid need to be observed to explain the biochemical behaviour of the materials using in joint replacement. Raman spectroscopy is a good technique to understand clearly the changes of the chemical structure, as Raman spectra contain the fingerprint of each compound that describes a specific chemical structure within the formed film. The spectroscopic differences provided for each of the protein lubricants before use and after experiment within the simulator of the ball on disc set up can explain the chemical reactions that are occurring within artificial joint replacement. Also, by observing the Raman spectra of lubricant film formed on the ball, one can address the chemical structural changes that are taking place due to bond breaking or formation within the proteins of synovial fluid. There could also be a possibility of heterogeneous catalysis process occurring among proteins within joint replacement in case of using CoCrMo alloy as a metal prosthesis. Furthermore, using mixtures of different synovial proteins in appropriate ratios as lubricants may show significant 
spectroscopic differences and thus it would be possible to understand the lubrication chemistry and mechanism of synovial liquid proteins within artificial prosthesis more explicitly.

\section{Acknowledgement}

This research was carried out under the project CEITEC 2020 (LQ1601) with financial support from the Ministry of Education, Youth and Sports of the Czech Republic under the National Sustainability Programme II.

\section{References}

Choudhury, D., Urban, F., Vrbka, M., Hartl, M. and Křupka, I. (2015) A novel tribological study on DLC-coated micro-dimpled orthopedics implant interface. Journal of the Mechanical Behavior of Biomedical Materials, 45, pp. 121-131.

Depciuch, J., Sowa-Kućma, M., Nowak, G., Dudek, D., Siwek, M., Styczeń, K. and Parlińska-Wojtan, M. (2016) Phospholipid-protein balance in affective disorders: Analysis of human blood serum using Raman and FTIR spectroscopy. A pilot study. Journal of Pharmaceutical and Biomedical Analysis,131, pp. 287-296.

Duong, C. T., Lee, J. H., Cho, Y., Nam, J. S., Kim, H. N., Lee, S. S. and Park, S. (2012) Effect of protein concentrations of bovine serum albumin and $\gamma$-globulin on the frictional response of a cobalt-chromium femoral head. Journal of Materials Science: Materials in Medicine, 23, 5, pp. 1323-1330.

Galandáková, A., Ulrichová, J., Langová, K., Hanáková, A., Vrbka, M., Hartl, M. and Gallo, J. (2017) Characteristics of synovial fluid required for optimization of lubrication fluid for biotribological experiments. Journal of Biomedical Materials Research Part B: Applied Biomaterials, 105, 6, pp. 1422-1431.

Han, X. X., Zhao, B. and Ozaki, Y. (2009) Surface-enhanced Raman scattering for protein detection. Analytical and Bioanalytical Chemistry, 394,7, pp. 1719-1727.

Hills, B. A. and Crawford, R. W. (2003) Normal and prosthetic synovial joints are lubricated by surface-active phospholipid. The Journal of Arthroplasty, 18, 4, pp. 499-505.

Mazzucco, D., Scott, R. and Spector, M. (2004) Composition of joint fluid in patients undergoing total knee replacement and revision arthroplasty: correlation with flow properties. Biomaterials, 25,18, pp. 4433-4445.

Nečas, D., Vrbka, M., Urban, F., Křupka, I. and Hartl, M. (2016) The effect of lubricant constituents on lubrication mechanisms in hip joint replacements. Journal of the Mechanical Behavior of Biomedical Materials, 55, pp. 295307.

Nečas, D., Vrbka, M., Urban, F., Gallo, J., Křupka, I. and Hartl, M. (2017) In situ observation of lubricant film formation in THR considering real conformity: The effect of diameter, clearance and material. Journal of the Mechanical Behavior of Biomedical Materials, 69, pp. 66-74.

Shanmukh, S., Jones, L., Driskell, J., Zhao, Y., Dluhy, R. and Tripp, R. A. (2006) Rapid and Sensitive Detection of Respiratory Virus Molecular Signatures Using a Silver Nanorod Array SERS Substrate. Nano Letter, 6,11, pp. 2630-2636.

Vrbka, M., Nečas, D., Křupka, I., Hartl, M., Urban, F. and Gallo, J. (2015) Visualization of lubricating films between artificial head and cup with respect to real geometry. Biotribology, 1, pp. 61-65.

Zou, S. and Weaver, M. J. (1998) Surface-Enhanced Raman Scattering on Uniform Transition-Metal Films: Toward a Versatile Adsorbate Vibrational Strategy for Solid-Nonvacuum Interfaces. Analytical Chemistry, 70,11, pp. 2387-2395 